\section{BMJ Open \\ Respiratory \\ Research}

\title{
Yield of EBUS-TBNA for the diagnosis of sarcoidosis: impact of operator and cytopathologist experience
}

\author{
Asma Navasakulpong, ${ }^{1,2}$ Manon Auger, ${ }^{3}$ Anne V Gonzalez ${ }^{1}$
}

To cite: Navasakulpong A, Auger M, Gonzalez AV. Yield of EBUS-TBNA for the diagnosis of sarcoidosis: impact of operator and cytopathologist experience. BMJ Open Resp Res 2016;3: e000144. doi:10.1136/ bmjresp-2016-000144

Received 13 May 2016 Revised 15 July 2016 Accepted 18 July 2016

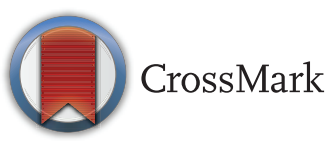

${ }^{1}$ Respiratory Epidemiology and Clinical Research Unit, Montreal Chest Institute, McGill University Health Centre, Montreal, Quebec, Canada

${ }^{2}$ Respiratory and Respiratory Critical Care Medicine, Faculty of Medicine, Prince of

Songkla University, Songkhla, Thailand

${ }^{3}$ Department of Pathology, McGill University and McGill University Health Centre, Montreal, Quebec, Canada

\section{Correspondence to} Dr Anne V Gonzalez; anne. gonzalez@mcgill.ca

\section{ABSTRACT}

Background: Studies have reported a high diagnostic yield of endobronchial ultrasound-guided transbronchial needle aspiration (EBUS-TBNA) for the diagnosis of sarcoidosis. We sought to review the yield of EBUS-TBNA for the diagnosis of sarcoidosis at our institution over time, and examine factors that may influence this yield.

Methods: Patients who underwent EBUS-TBNA for suspected sarcoidosis between December 2008 and November 2011 were identified. EBUS was performed without rapid on-site evaluation (ROSE) of samples. The final diagnosis was based on the results of all invasive diagnostic procedures and/or clinical followup. Logistic regression analysis was used to examine the effect of various factors on yield.

Results: 43 patients underwent 45 EBUS-TBNA procedures for suspected sarcoidosis. A total of 115 lymph nodes were sampled. The $21 \mathrm{G}$ needle was used in $51 \%$ of procedures. The mean number of lymph node stations sampled was 2.6 (SD 0.7 ) and the mean number of needle passes per procedure was 7.8 (SD 2.0). Non-necrotising granulomatous inflammation was detected in EBUS-TBNA samples from $34 / 45(76 \%)$ procedures. The overall diagnostic yield increased to $36 / 45(80 \%)$ following a cytopathology review for this study. Needle gauge, number of lymph node stations sampled and number of needle passes were not associated with diagnostic yield. The yield of EBUS-TBNA increased significantly after the first 15 procedures performed for suspected sarcoidosis; the 2 additional cases diagnosed after the cytopathology review were part of this early experience.

Conclusions: EBUS-TBNA is a valuable technique for the diagnosis of sarcoidosis when performed without ROSE. The yield of the procedure improved significantly over time, based on operator and cytopathologist experience.

\section{INTRODUCTION}

Sarcoidosis is a benign, multisystem inflammatory disorder of unknown aetiology that involves the lungs in more than 90\% of patients. The diagnosis of sarcoidosis is based on a compatible clinical and radiographic

\section{KEY MESSAGES}

EBUS-TBNA is a valuable technique for the diagnosis of sarcoidosis.

There is a significant early learning curve to the successful cytopathological diagnosis of sarcoidosis using EBUS-TBNA, which is partly shared with the cytopathologists.

picture, with demonstration of nonnecrotising granulomatous inflammation on tissue biopsy, in the absence of inciting organisms or particles. ${ }^{1}$

Endobronchial ultrasound-guided transbronchial needle aspiration (EBUS-TBNA) is a minimally invasive technique that allows sampling of mediastinal and hilar nodes. The role of EBUS-TBNA in the diagnosis and staging of lung cancer is well established. ${ }^{2}{ }_{3}$ Recent studies have reported a high diagnostic yield of EBUS-TBNA for the diagnosis of sarcoidosis. In a randomised trial of EBUS-TBNA versus conventional TBNA for the diagnosis of sarcoidosis, Tremblay $e t \quad a t^{4}$ reported a significantly higher diagnostic yield of EBUS-TBNA $(83.3 \%$ vs $53.8 \%, \quad \mathrm{p}<0.05)$. A systematic review and meta-analysis reported a pooled diagnostic accuracy of $79 \%$ (95\% CI $71 \%$ to $86 \%$ ) of EBUS-TBNA for the diagnosis of sarcoidosis. ${ }^{5}$ The clinical factors associated with successful demonstration of nonnecrotising granulomatous inflammation in EBUS-TBNA samples have not been clearly established. ${ }^{6}$

We sought to review the diagnostic yield of EBUS-TBNA for the diagnosis of sarcoidosis at our institution. The perception of initially disappointing EBUS-TBNA results in patients with suspected sarcoidosis led us to examine diagnostic yield over time, in addition to other factors that may influence this yield. These results have been previously presented at the American College of Chest Physicians meeting. ${ }^{7}$ 


\section{METHODS}

This was a single-centre retrospective study. A prospective list of all patients who underwent EBUS-TBNA at the Montreal Chest Institute has been maintained as part of a quality control initiative. Patients who underwent EBUS-TBNA for suspected sarcoidosis between December 2008 and November 2011 were identified from this prospectively maintained list. Specifically, the original indication for the procedure was reviewed. Patients referred for investigation of suspected sarcoidosis, or for the workup of adenopathy of unknown aetiology, in whom sarcoidosis was high on the differential diagnosis, were included. Patients referred for diagnosis and/or staging of lung cancer or another malignancy were excluded. The final diagnosis was based on the results of all invasive diagnostic procedures performed and/or clinical follow-up. The study was approved by the McGill University Health Centre Research Ethics Board.

Real-time EBUS-TBNA was performed using a dedicated flexible bronchoscope with an integrated ultrasound transducer (BF-UC160F, Olympus Canada). The procedures were performed by a single operator supervising different trainees. Patients received moderate sedation with midazolam (or propofol) and fentanyl. The number of lymph node stations sampled and number of aspirates per node were at the discretion of the operator. Targeted lymph nodes were punctured with either the 21 or 22 gauge (G) EBUS-guided TBNA needles (NA-201SX-4021, NA-201SX-4022), also at the discretion of the main operator. EBUS was performed in the absence of rapid on-site evaluation (ROSE) of samples by a cytopathologist. The aspirated specimens from each lymph node station were flushed into containers filled with small amounts of saline, to which CytoLyte (methanol-water solution) was added postprocedure. Specimens were then processed in the cytopathology laboratory. The samples first underwent centrifugation. If no pellet was present after centrifugation, cytospins were prepared; smears with or without a cell block were prepared if a pellet was visible, depending on the size of the pellet.

The demographic characteristics of all patients investigated for suspected sarcoidosis were tabulated, including patient age, gender, sarcoidosis stage and lymph node size based on CT and/or EBUS measurements. Procedures were recorded in chronological order. The following procedure characteristics were extracted: number of lymph node stations sampled, number of needle passes performed, anatomic location of the lymph node stations sampled, needle gauge (21 vs $22 \mathrm{G}$ ), type of sedation used and complications. All bronchoscopy samples taken in addition to the EBUS-guided needle aspirates were documented. The results of EBUS-TBNA and all additional invasive procedures performed were recorded. The diagnosis of sarcoidosis was considered confirmed if the clinicoradiological findings were supported by the cytological or histopathological presence of non-necrotising granulomatous inflammation. Other possible causes of granulomatous inflammation were examined by reviewing patient histories and microbiology results (culture of EBUS-TBNA or other specimens, where available).

All the cytopathology specimens of patients who underwent EBUS-TBNA for suspected sarcoidosis were reviewed by a cytopathologist (MA) for the purpose of this research project. The results of this study review were compared with the diagnoses originally established by the cytopathologists involved in the cases. The contribution of slides versus cell block to establishing the presence of non-necrotising granulomatous inflammation was examined.

The database was constructed using Excel 2007 (Microsoft Office). Categorical variables are presented as the proportion of patients or procedures performed. For continuous variables, mean $\pm \mathrm{SD}$ are shown. The overall diagnostic yield of EBUS-TBNA in patients with suspected sarcoidosis was calculated before and after the study cytopathology review; diagnostic yield was also estimated over time (every 15 procedures). Univariate and multivariate logistic regression analysis was used to assess the factors associated with a positive diagnostic yield of EBUS-TBNA in patients with suspected sarcoidosis. Statistical analyses were performed using SAS V.9.1. The diagnostic yield of EBUS-TBNA after every 15 procedures performed for suspected sarcoidosis was plotted using the GraphPad Prism.

\section{RESULTS}

A total of 315 EBUS-TBNA procedures were performed over the 35-month study period. The most frequent indication was lung cancer diagnosis and staging. A total of 45 procedures were performed in 43 patients for suspected sarcoidosis. Patient and procedure characteristics are detailed in table 1 . The mean age of patients was 50 years and most patients had radiographic stage I or II disease. A total of 115 lymph nodes were sampled. On

\begin{tabular}{ll} 
Table $1 \quad$ Baseline characteristics & \\
\hline Patient characteristics (N=43) & $50.1(13.7)$ \\
Mean age, in years (SD) & 55.8 \\
$\begin{array}{l}\text { Male gender (\%) } \\
\text { Sarcoidosis stage (\%) }\end{array}$ & 41.9 \\
1 & 55.8 \\
2 & 2.3 \\
3 & \\
Procedure characteristics (N=45) & $2.6(0.7)$ \\
Mean number of lymph node stations & \\
sampled (SD) & $7.8(2.0)$ \\
Mean number of needle passes (SD) & \\
Location of lymph node stations sampled (\%) & 13.3 \\
$\quad$ Mediastinal nodes only & 86.7 \\
$\quad$ Hilar and mediastinal nodes & 51.1 \\
Needle gauge, 21 (\%) & \\
\hline Values are shown as mean (SD) or proportions.
\end{tabular}


average, 2.6 lymph node stations (SD 0.7) were sampled, with a mean of 7.8 needle passes (SD 2.0) per procedure. In the majority of procedures, both hilar and mediastinal nodes were sampled. The $21 \mathrm{G}$ needle was used in approximately half the cases while the remainder was performed using the $22 \mathrm{G}$ needle.

Non-necrotising granulomatous inflammation was detected in EBUS-TBNA samples from 34 procedures. The overall yield of EBUS-TBNA for the diagnosis of sarcoidosis was $76 \%$ (34/45 procedures). Non-necrotising granulomatous inflammation was detected in samples other than the EBUS-TBNA in four patients: endobronchial biopsies (taken at the time of the EBUS procedure) and videoassisted thorascopic surgery (VATS) established the pathological diagnosis in two patients, while two patients underwent mediastinoscopy (figure 1). In the remaining five patients for whom no granulomatous inflammation could be identified in the pathological material, follow-up confirmed the clinical impression of sarcoidosis. Culture results were available in over $60 \%$ of patients and negative; mycobacterial and fungal stains of EBUS-TBNA slides showing granulomata were done systematically and were negative in all cases (data not shown).

Non-necrotising granulomatous inflammation was detected in samples from an additional two EBUS-TBNA procedures following the study cytopathology review. The overall yield of EBUS-TBNA for the diagnosis of sarcoidosis thus increased to $80 \%$ (36/45 procedures). Both cytology slides and cell block had a role in establishing the diagnosis of sarcoidosis. However, in a third of cases, non-necrotising granulomatous inflammation was detected in the cell block only. A cell block was available in $42 / 45$ procedures (93.3\%), while slides were available in all cases. The additional diagnoses established at the study cytopathology review were based on slides alone (table 2).

Logistic regression analysis was used to examine potential factors associated with a positive diagnosis of EBUS-TBNA in patients with suspected sarcoidosis. Having performed over 15 procedures for suspected sarcoidosis was the only factor associated with a positive diagnostic yield on univariate analysis (table 3). Needle gauge, number of lymph node stations sampled, lymph node size and total number of needle passes performed did not significantly impact diagnostic yield. When adjusted for the number of lymph node stations sampled and total number of needle passes, having performed over 15 EBUS-TBNA procedures for suspected sarcoidosis remained significantly associated with diagnostic yield (table 4). The effect of the 21 and $22 \mathrm{G}$ needles could not be examined in this multivariate model because the first 18 procedures were performed with the $22 \mathrm{G}$ needle (when the $21 \mathrm{G}$ needle was not yet available).

The diagnostic yield of EBUS-TBNA for sarcoidosis, before and after the study cytopathology review was plotted according to the number of procedures performed specifically for suspected sarcoidosis; the total number of procedures performed during the same time period is also indicated (figure 2). The diagnostic yield of EBUS-TBNA increased to over $90 \%$ after the first 15 procedures performed for suspected sarcoidosis (when over 100 EBUS-TBNA procedures had been performed locally by the primary operator). The two additional

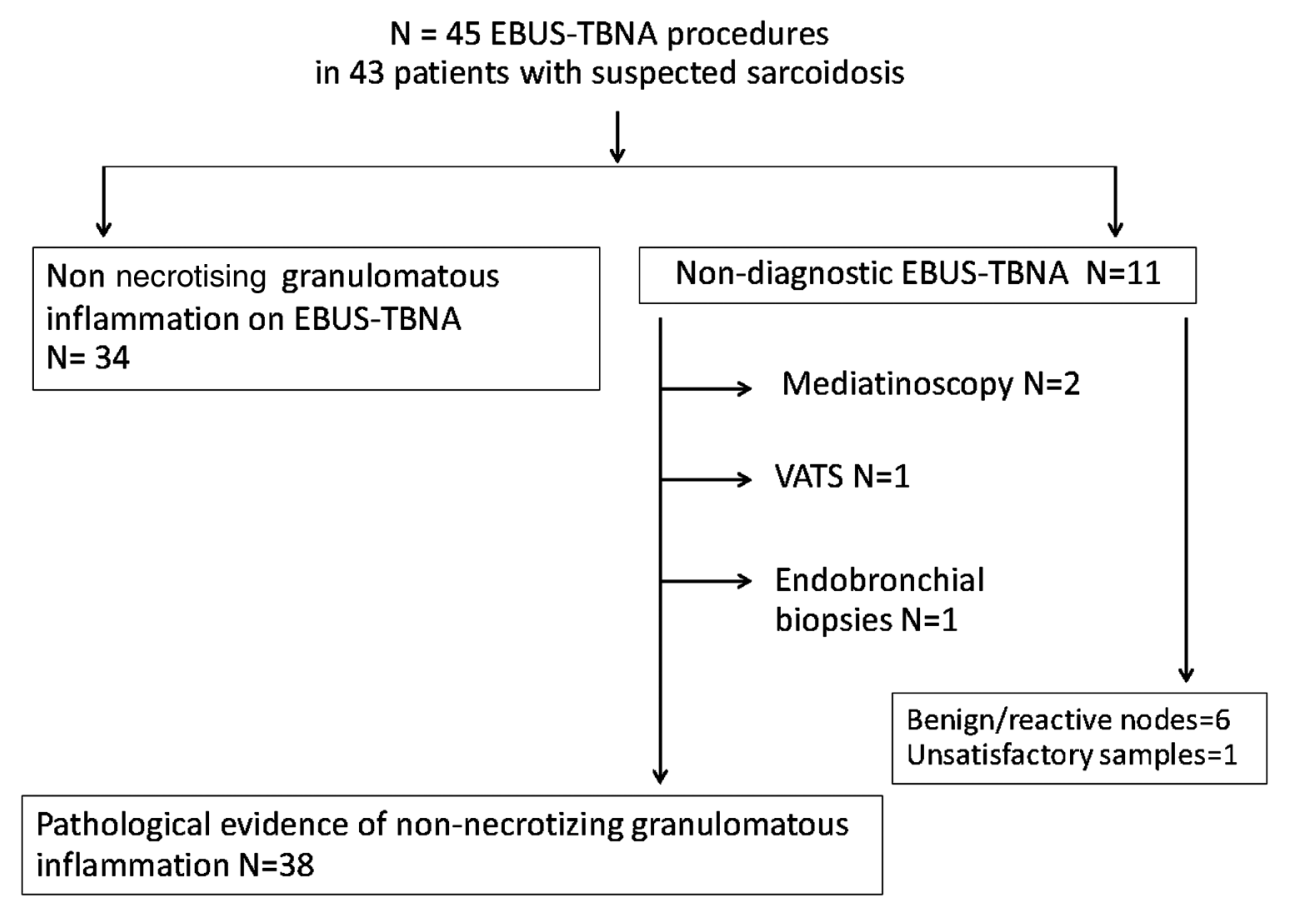

Figure 1 Endobronchial ultrasound-guided transbronchial needle aspiration (EBUS-TBNA) and other diagnostic procedures in patients with suspected sarcoidosis. 
Table 2 Diagnostic yield of endobronchial ultrasound-guided transbronchial needle aspiration for suspected sarcoidosis, before and after the research cytopathology review

\begin{tabular}{|c|c|c|}
\hline & Initial diagnostic yield & $\begin{array}{l}\text { Diagnostic yield } \\
\text { following the } \\
\text { cytopathology review }\end{array}$ \\
\hline \multicolumn{3}{|l|}{ Per procedure $(\mathrm{N}=45)$} \\
\hline Overall diagnostic yield & $34 / 45(75.6)$ & $36 / 45(80)$ \\
\hline Diagnosis from cell block only, where available $(\mathrm{N}=42)$ & 14/42 (33.3) & $14 / 42(33.3)$ \\
\hline Diagnosis from slides and cell block, where available $(\mathrm{N}=42)$ & $19 / 42(4.5)$ & $19 / 42(4.5)$ \\
\hline Diagnosis from slides alone (slides available in all 45 patients) & $1 / 45(2.2)$ & $3 / 45(6.7)$ \\
\hline \multicolumn{3}{|l|}{ Per lymph node $(\mathrm{N}=115)$} \\
\hline Overall diagnostic yield & $68 / 115(59.1)$ & $73 / 115(63.5)$ \\
\hline Diagnosis from cell block only, where available $(\mathrm{N}=89)$ & $37 / 89(41.6)$ & $37 / 89(41.6)$ \\
\hline Diagnosis from slides and cell block, where available $(\mathrm{N}=89)$ & $30 / 89(33.7)$ & $31 / 89(34.8)$ \\
\hline Diagnosis from slides alone (slides available in all 115 nodes) & $1 / 115(0.9)$ & $5 / 115(4.3)$ \\
\hline
\end{tabular}

Table 3 Univariate logistic regression analysis of potential factors associated with a positive diagnosis of endobronchial ultrasound-guided transbronchial needle aspiration for suspected sarcoidosis

\begin{tabular}{|c|c|c|}
\hline & OR & $95 \% \mathrm{Cl}$ \\
\hline Needle gauge (No 21 vs No 22 ) & 2.50 & 0.54 to 11.59 \\
\hline Total number of needle passes & 0.95 & 0.65 to 1.38 \\
\hline $\begin{array}{l}\text { Total number of needle passes, } \\
>5 \text { vs } \leq 5 \text { passes }\end{array}$ & 1.38 & 0.13 to 15.03 \\
\hline $\begin{array}{l}\text { Number of lymph node stations } \\
\text { sampled }\end{array}$ & 0.55 & 0.18 to 1.73 \\
\hline $\begin{array}{l}\text { Number of lymph node stations } \\
\text { sampled, }>2 \text { vs } \leq 2 \text { stations }\end{array}$ & 0.36 & 0.07 to 1.96 \\
\hline Lymph node size, $\geq 2$ vs $<2 \mathrm{~cm}$ & 0.57 & 0.13 to 2.49 \\
\hline $\begin{array}{l}\text { Number of procedures performed, } \\
>15 \text { vs } \leq 15 \text { procedures }\end{array}$ & 6.00 & 1.24 to 29.07 \\
\hline
\end{tabular}

cases diagnosed at the time of the study cytopathology review were part of the early experience of EBUS-TBNA for suspected sarcoidosis.

\section{DISCUSSION}

EBUS-TBNA has an established role for the diagnosis and staging of lung cancer. Minimally invasive needle techniques, including EBUS and EUS (endoscopic ultrasound)-guided needle aspiration, are now the recommended test of first choice for mediastinal staging in patients with lung cancer. ${ }^{3}$ While EBUS-TBNA is also being used increasingly for the diagnosis of benign conditions, the diagnostic yield of the procedure may be more variable. In this retrospective study of our early experience with EBUS-TBNA in patients with suspected sarcoidosis, the overall diagnostic yield was $76 \%$ for the detection of non-necrotising granulomatous inflammation in accessible hilar and/or mediastinal lymph nodes. A significant early learning curve was detected, with diagnostic yield increasing to $90 \%$ after the first 15 procedures performed for suspected sarcoidosis.

Several studies have reported on the efficacy of EBUS-TBNA for the diagnosis of pulmonary sarcoidosis. Garwood et $a l,{ }^{8}$ using ROSE of needle aspirate samples, reported that EBUS-TBNA had a sensitivity of $85 \%$ for the diagnosis of pulmonary sarcoidosis. In the study of Wong et $a l^{9}$ EBUS-TBNA demonstrated non-caseating epithelioid cell granulomata in 56/61 (91.8\%) patients with a final diagnosis of sarcoidosis. ROSE was also available to these authors and, as a result, a mean of only 1.2 lymph nodes were sampled per patient. In a smaller series of patients, Oki et $a l^{10}$ reported that EBUS-TBNA had a sensitivity of $93 \%$ for the diagnosis of sarcoidosis in the absence of ROSE. In a retrospective review of 35 patients with stage I or II sarcoidosis, Nakajima and colleagues compared EBUS-TBNA with other bronchoscopic sampling methods for the diagnosis of sarcoidosis. The diagnostic accuracy for EBUS-TBNA with ROSE was significantly better than transbronchial biopsy,

Table 4 Multivariate logistic regression analysis of potential factors associated with a positive diagnosis of endobronchial ultrasound-guided transbronchial needle aspiration for suspected sarcoidosis

\begin{tabular}{|c|c|c|c|c|}
\hline & OR $^{*}$ & $95 \% \mathrm{Cl}$ & OR $^{*}$ & $95 \% \mathrm{Cl}$ \\
\hline Needle gauge (No 21 vs No 22) & & & 2.10 & 0.39 to 11.23 \\
\hline Total number of needle passes & 0.75 & 0.40 to 1.40 & 1.03 & 0.63 to 1.66 \\
\hline Number of lymph node stations sampled & 1.17 & 0.24 to 5.86 & 0.61 & 0.14 to 2.59 \\
\hline Number of procedures performed, $>15$ vs $\leq 15$ procedures & 9.37 & 1.18 to 74.27 & & \\
\hline
\end{tabular}

*Adjusted for the other factors in the same column. 


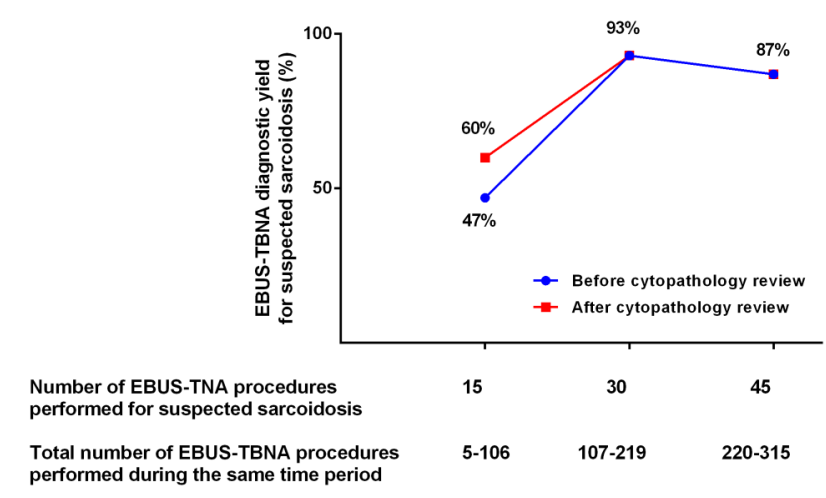

Figure 2 Evolution of endobronchial ultrasound-guided transbronchial needle aspiration (EBUS-TBNA) diagnostic yield for suspected sarcoidosis, before and after the research cytopathology review.

or a bronchoalveolar lavage $\mathrm{CD} 4 / \mathrm{CD} 8$ ratio $>3.5$ $(91.4 \%, 40 \%$ and $65.7 \%$, respectively: $\mathrm{p}<0.001) .{ }^{11}$

Tremblay et al performed the first randomised controlled trial (RCT) of conventional versus EBUS-guided TBNA in patients with suspected sarcoidosis. The diagnostic yield of EBUS-TBNA for stage I and II sarcoidosis was superior to that of conventional TBNA $(83.3 \%$ vs $53.8 \%, \mathrm{p}<0.05$; an absolute increase of $29.5 \%, 95 \%$ CI $8.6 \%$ to $55.4 \%) .{ }^{4}$ A systematic review and meta-analysis on the role of EBUS-TBNA for the diagnosis of sarcoidosis reported a pooled diagnostic accuracy of $79 \%(95 \%$ CI $71 \%$ to $86 \%) .{ }^{5}$ The yield was not significantly greater in studies that employed ROSE. All studies included had used the $22 \mathrm{G}$ needle. There was evidence of significant clinical and statistical heterogeneity, as well as publication bias. A recently published guideline on technical aspects of EBUS-TBNA included an updated search on the efficacy of EBUS-TBNA for the diagnosis of sarcoidosis, with a pooled diagnostic accuracy of $78.2 \%$ (95\% CI $75.6 \%$ to $80.4 \%)^{12}$

While EBUS-TBNA appears to be a sensitive and useful tool for the diagnosis of sarcoidosis, few studies have examined the factors associated with a positive diagnostic yield. In the prospective study of Garwood et $a l,{ }^{8}$ the yield per needle pass exceeded $80 \%$ at five passes, with no further increase beyond seven passes. Using the combined data from their RCT of conventional versus EBUS-guided TBNA, Chee et at examined the relative importance of cytological preparations and factors influencing the diagnostic yield in patients with suspected sarcoidosis. Liquid-based cytology and cell blocks were equally important in identifying granulomatous inflammation. There was no correlation between the number of aspirates per node, lymph node location or size and diagnostic yield. In the current study, nonnecrotising granulomata were only detected in the cell block material for a third of procedures. A cell block was available in $93 \%$ of procedures (cell block unavailable in one procedure performed with the $21 \mathrm{G}$ and two performed with the $22 \mathrm{G}$ needles, respectively). The number of lymph node stations sampled and number of needle passes were not predictive of EBUS-TBNA diagnostic yield in suspected sarcoidosis.

We were unable to examine the impact of the 21 and $22 \mathrm{G}$ needles on diagnostic yield using multivariate regression analysis, because the first 18 EBUS-TBNA procedures were all performed using the $22 \mathrm{G}$ needle (prior to the $21 \mathrm{G}$ needle becoming available). However, no association was detected between needle gauge and diagnostic yield in the univariate analysis. Nakajima and colleagues sampled 45 lesions using both the 21 and $22 \mathrm{G}$ needles, and reported no difference in diagnostic yield. The $21 \mathrm{G}$ needle was associated with better preservation of histological structure, but more blood contamination. Granulomatous changes suspicious for sarcoidosis were detected in eight sites. Interestingly, non-necrotising granulomata tended to be more preserved in samples obtained with the $22 \mathrm{G}$, rather than $21 \mathrm{G}$ needle $(\mathrm{p}=0.0695) .{ }^{13}$ Using data from the American College of Chest Physicians (ACCP) AQuIRE Registry, Yarmus et $a l^{14}$ reviewed sample adequacy and diagnostic yield in 1235 EBUS-TBNA procedures, and found no difference between the 21 and $22 \mathrm{G}$ needles.

The existence of an EBUS-TBNA operator learning curve is well established, although its length is far from clear. Wahidi et $a l^{15}$ examined the EBUS-TBNA learning curve of general pulmonary fellows and reported that an average of 13 procedures was required to perform the first independent successful procedure. Various authors have reported that diagnostic accuracy continued to improve after $50^{16} 100^{17}$ and even $140^{18}$ procedures. Stather $e t a l^{19}$ assessed the EBUS learning curve of nine interventional pulmonary fellows using computer simulator testing; significant variation was detected, with ongoing improvement in EBUS skill even after 200 clinical cases. In this study, the diagnostic yield of EBUS-TBNA increased to over $90 \%$ after the first 15 procedures performed for suspected sarcoidosis, at a time when over 100 procedures had been performed by the main operator at the institution. The two additional cases diagnosed at the cytopathology review were part of the early experience of EBUS-TBNA in patients with suspected sarcoidosis (first 15 procedures). This suggests that part of the early learning curve in sarcoidosis is shared with the cytopathologists.

In the RCT of Tremblay et al, the diagnostic yield for conventional and EBUS-TBNA increased significantly following review by a cytopathologist specialised in lung diseases. ${ }^{4}$ Skov and colleagues assessed the diagnoses established on EBUS and EUS samples by pathologists who had variable experience with these types of samples. The reproducibility of diagnoses was found to be excellent among experienced pathologists; pathologists with general experience but little experience with EBUS or EUS samples had a steep learning curve. ${ }^{20}$ In this study, cell blocks had an important role, with nonnecrotising granulomatous inflammation being 
identified in cell block only in $1 / 3$ of cases. Granulomata were detected on smears in the two additional diagnoses established by the cytopathology review. Granulomata are more difficult to recognise on smears than in cell block material. As cytopathologists gain more experience with such cases, they may feel more secure diagnosing granulomata, particularly if present on smears only. In a narrative review of EBUS-TBNA applications in chest disease, Medford and colleagues commented on the smaller evidence base in benign mediastinal disease, and suggested that benign diagnoses may be more dependent on local cytopathology services. $^{21}$

In conclusion, EBUS-TBNA is a valuable technique for the diagnosis of sarcoidosis when performed in the absence of ROSE. There is a significant early learning curve to the successful cytopathological diagnosis of sarcoidosis using EBUS-TBNA. This suggests that clinicians should expect improvement in the yield of EBUS-TBNA in patients with suspected sarcoidosis once both the bronchoscopist and cytopathologist gain more familiarity with the procedures and specimens, respectively.

Acknowledgements The authors wish to thank Ms Pei Zhi Li for her assistance with statistical analyses.

Funding Fonds de Recherche du Québec-Santé.

Competing interests None declared.

Ethics approval The study was approved by the McGill University Health Centre Research Ethics Board.

Provenance and peer review Not commissioned; externally peer reviewed.

Data sharing statement No additional data are available.

Open Access This is an Open Access article distributed in accordance with the Creative Commons Attribution Non Commercial (CC BY-NC 4.0) license, which permits others to distribute, remix, adapt, build upon this work noncommercially, and license their derivative works on different terms, provided the original work is properly cited and the use is non-commercial. See: http:// creativecommons.org/licenses/by-nc/4.0/

\section{REFERENCES}

1. Costabel U, Hunninghake GW. ATS/ERS/WASOG statement on sarcoidosis. Sarcoidosis Statement Committee. American Thoracic Society. European Respiratory Society. World Association for Sarcoidosis and Other Granulomatous Disorders. Eur Respir J 1999;14:735-7.

2. Adams K, Shah PL, Edmonds L, et al. Test performance of endobronchial ultrasound and transbronchial needle aspiration biopsy for mediastinal staging in patients with lung cancer: systematic review and meta-analysis. Thorax 2009;64:757-62.

3. Silvestri GA, Gonzalez AV, Jantz MA, et al. Methods for staging non-small cell lung cancer: diagnosis and management of lung cancer, 3rd ed: American College of Chest Physicians evidence-based clinical practice guidelines. Chest 2013;143: e211S-250S.

4. Tremblay A, Stather DR, Maceachern $\mathrm{P}$, et al. A randomized controlled trial of standard vs endobronchial ultrasonography-guided transbronchial needle aspiration in patients with suspected sarcoidosis. Chest 2009;136:340-6.

5. Agarwal R, Srinivasan A, Aggarwal AN, et al. Efficacy and safety of convex probe EBUS-TBNA in sarcoidosis: a systematic review and meta-analysis. Respir Med 2012;106:883-92.

6. Chee A, Khalil M, Stather DR, et al. Cytologic assessment of endobronchial ultrasound-guided transbronchial needle aspirates in sarcoidosis. J Bronchol Intervent Pulmonol 2012;19:24-8.

7. Navasakulpong A, Auger M, Gonzalez AV. Determinants of the yield of endobronchial ultrasound-guided transbronchial needle aspiration (EBUS-TBNA) for the diagnosis of sarcoidosis. Chest 2013;144:818A

8. Garwood S, Judson MA, Silvestri G, et al. Endobronchial ultrasound for the diagnosis of pulmonary sarcoidosis. Chest 2007;132:1298-304.

9. Wong M, Yasufuku K, Nakajima T, et al. Endobronchial ultrasound: new insight for the diagnosis of sarcoidosis. Eur Respir $J$ 2007;29:1182-6.

10. Oki M, Saka H, Kitagawa C, et al. Real-time endobronchial ultrasound-guided transbronchial needle aspiration is useful for diagnosing sarcoidosis. Respirology 2007;12:863-8.

11. Nakajima T, Yasufuku K, Kurosu K, et al. The role of EBUS-TBNA for the diagnosis of sarcoidosis-comparisons with other bronchoscopic diagnostic modalities. Respir Med 2009;103:1796-800.

12. Wahidi MM, Herth F, Yasufuku K, et al. Technical aspects of endobronchial ultrasound guided transbronchial needle aspiration: CHEST guideline and expert panel report. Chest 2016;149:816-35.

13. Nakajima T, Yasufuku K, Takahashi R, et al. Comparison of 21-gauge and 22-gauge aspiration needle during endobronchial ultrasound-guided transbronchial needle aspiration. Respirology 2011;16:90-4

14. Yarmus LB, Akulian J, Lechtzin N, et al. Comparison of 21-gauge and 22-gauge aspiration needle in endobronchial ultrasound-guided transbronchial needle aspiration: results of the American College of Chest Physicians Quality Improvement Registry, Education, and Evaluation Registry. Chest 2013;143:1036-43.

15. Wahidi MM, Hulett C, Pastis N, et al. Learning experience of linear endobronchial ultrasound among pulmonary trainees. Chest 2014:145:574-8.

16. Steinfort DP, Hew MJ, Irving LB. Bronchoscopic evaluation of the mediastinum using endobronchial ultrasound: a description of the first 216 cases carried out at an Australian tertiary hospital. Intern Med $J$ 2011;41:815-24.

17. Fernandez-Villar A, Leiro-Fernandez V, Botana-Rial M, et al. The endobronchial ultrasound-guided transbronchial needle biopsy learning curve for mediastinal and hilar lymph node diagnosis. Chest 2012;141:278-9.

18. Medford AR. Learning curve for endobronchial ultrasound-guided transbronchial needle aspiration. Chest 2012;141:1643; author reply 1643-4.

19. Stather DR, Chee A, MacEachern P, et al. Endobronchial ultrasound learning curve in interventional pulmonary fellows. Respirology 2015;20:333-9.

20. Skov BG, Baandrup U, Jakobsen GK, et al. Cytopathologic diagnoses of fine-needle aspirations from endoscopic ultrasound of the mediastinum: reproducibility of the diagnoses and representativeness of aspirates from lymph nodes. Cancer 2007;111:234-41.

21. Medford AR, Bennett JA, Free CM, et al. Endobronchial ultrasound-guided transbronchial needle aspiration (EBUS-TBNA): applications in chest disease. Respirology 2010;15:71-9. 